\title{
Endoscopic full-thickness resection of a nonlifting adenoma in an ileal pouch using an over-the-scope full-thickness resection device
}

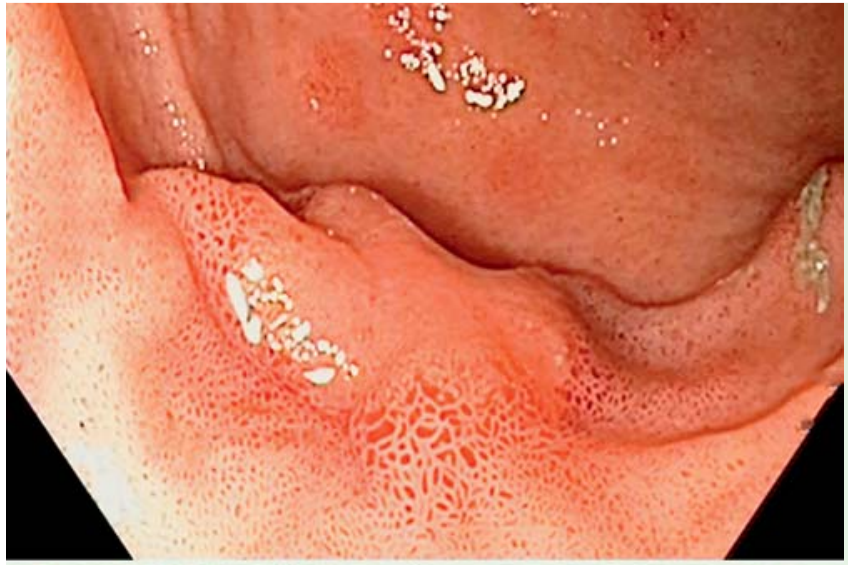

Fig. 1 Nonlifting Paris 0 -IIb lesion, located $3 \mathrm{~cm}$ proximal to the ileo-anal anastomosis on the dorsal wall of the J-pouch. No malignant characteristics were seen on narrow-band imaging (Narrow-band imaging International Colorectal Endoscopic [NICE] classification type 2).
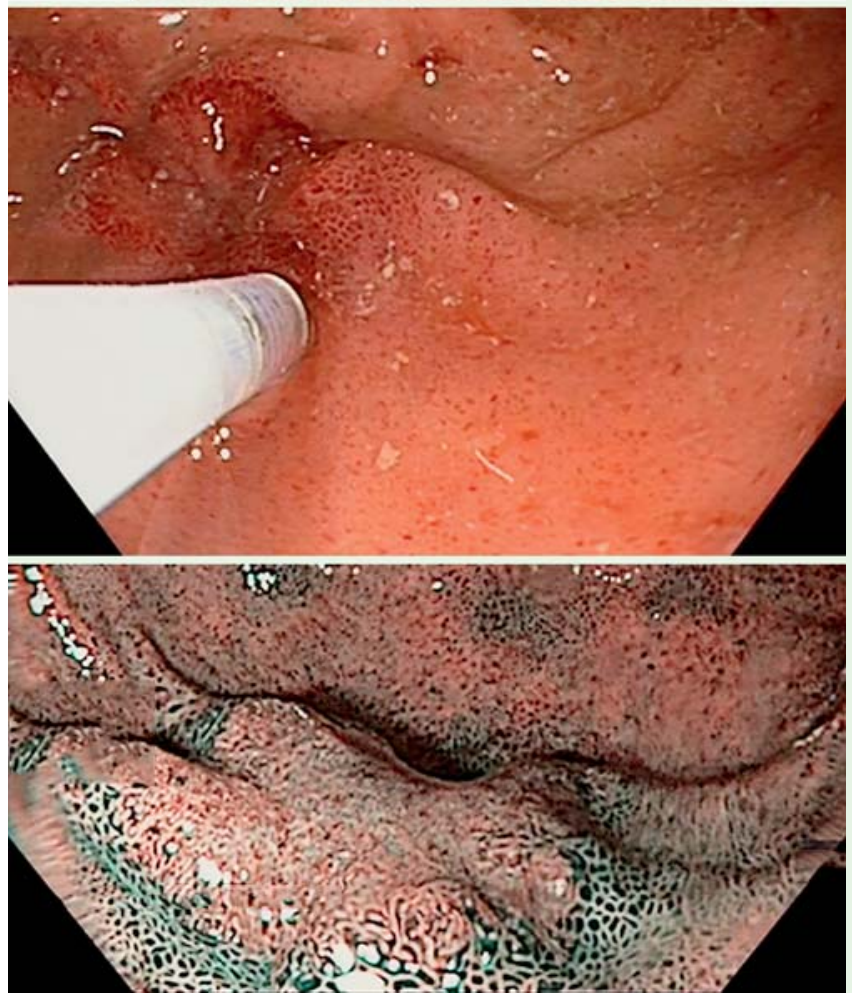

A 34-year-old man with familial adenomatous polyposis and ileo-anal pouch anastomosis was referred for treatment of a nonlifting Paris 0-IIb lesion. The lesion was located $3 \mathrm{~cm}$ proximal to the ileo-anal anastomosis on the dorsal wall of the J-pouch. No malignant characteristics were seen on narrow-band imaging (Narrow-band imaging International Colorectal Endoscopic [NICE] classification type 2) ( $\bullet$ Fig. 1).

Full-thickness resection with an over-thescope full-thickness resection device
(FTRD) was proposed ( $\bullet$ Video 1 ). After the lesion had been delineated using coagulation marks, the endoscope, together with the mounted FTRD, was inserted into the pouch. In the absence of mobile surrounding structures in this distally located lesion, it was considered safe to perform the full-thickness resection without the use of the tissue grasper that was incorporated into the FTRD. Gentle suctioning was used to mobilize the lesion into the cap ( $\bullet$ Fig. 2 ), after which the over-the-scope clip (OTSC) was deployed

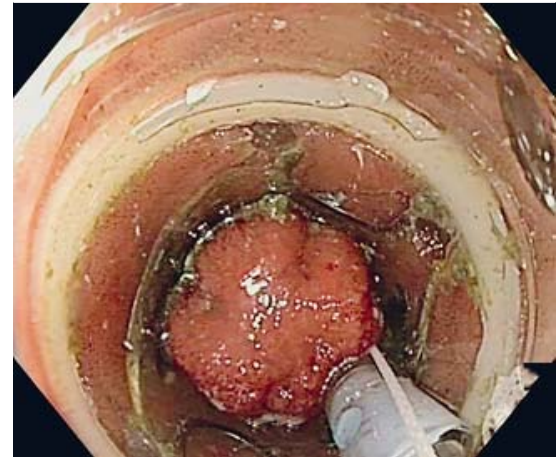

Fig. 2 Gentle suctioning mobilized the lesion into the cap of the full-thickness resection device.

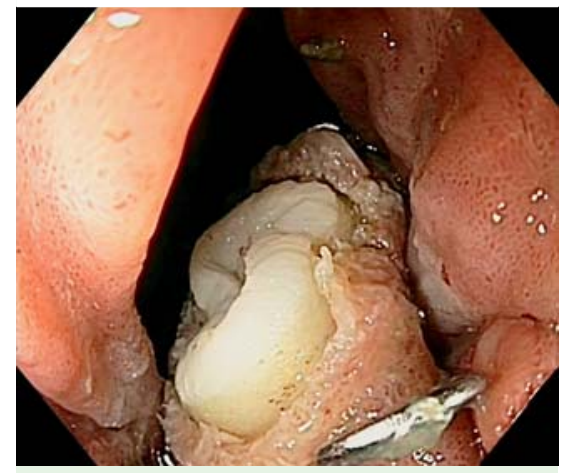

Fig. 3 The muscular layer was grasped into the over-the-scope-clip, thus preserving the integrity of the intestinal wall. The resection was macroscopically complete.

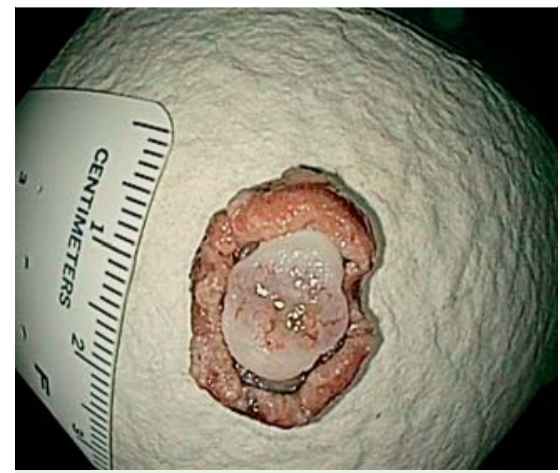

Fig. 4 The serosa was macroscopically visible on the resection specimen.

and immediate resection using the preloaded snare was performed. The resection was macroscopically complete. The muscular layer was grasped into the OTSC, thus preserving the integrity of the intestinal wall ( $\bullet$ Fig.3). The serosa was 


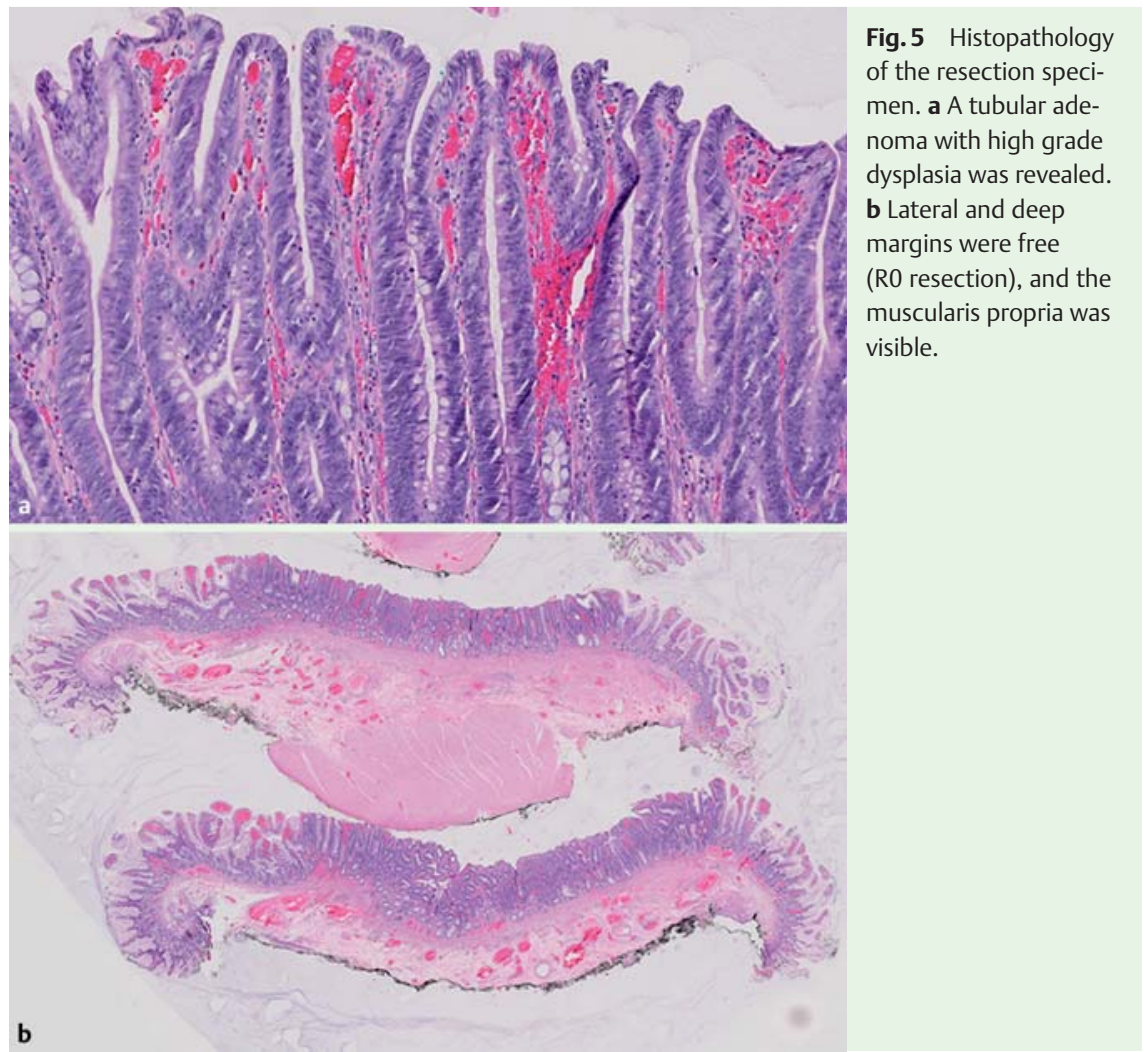

also visible on the resection specimen ( Fig.4). The size of the lesion was $20 \times$ $14 \mathrm{~mm}$. No post-procedural complications were noted.

Histopathology revealed a tubular adenoma with high grade dysplasia. Lateral and deep margins were free, and the presence of the muscularis propria was confirmed (๑ Fig. 5).

To our knowledge, this is the first report of endoscopic full-thickness resection performed in an ileal pouch using the overthe-scope FTRD. Endoscopic full-thickness resection may be a valuable therapeutic tool for nonlifting lesions in the lower gastrointestinal tract [1].

The probability of submucosal invasion in nonlifting lesions is high [2]. As an alternative to endoscopic submucosal dissection, the FTRD allows en bloc resection of lesions $<25 \mathrm{~mm}$ and histopathological evaluation of submucosal, muscular, and serosal layers. This allows accurate assess- ment of the risk of lymph node involvement and of the need for additional surgery.

In summary, use of the FTRD can provide an adequate full-thickness resection as well as a reliable closure mechanism, even in patients with ileo-anal pouch anastomosis.

Endoscopy_UCTN_Code_TTT_1AQ_2AD

\section{Competing interests: None}

\section{Christophe Snauwaert ${ }^{1}$, Anne Jouret- Mourin $^{2}$, Hubert Piessevaux ${ }^{1}$}

${ }^{1}$ Department of Gastroenterology and Hepatology, Cliniques Universitaires Saint-Luc, Université catholique de Louvain, Brussels, Belgium

${ }^{2}$ Department of Pathology, Cliniques Universitaires Saint-Luc, Université catholique de Louvain, Brussels, Belgium

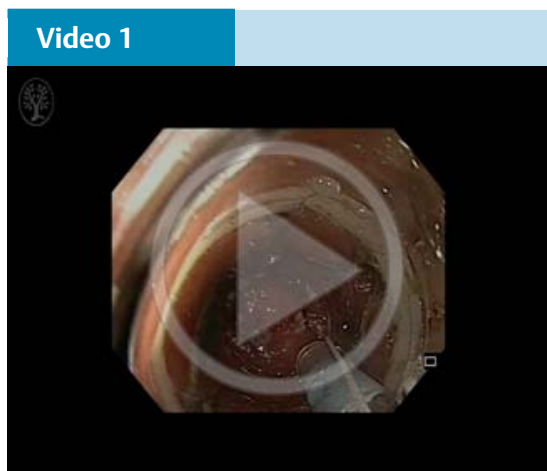

Endoscopic full-thickness resection of a nonlifting adenoma in an ileal pouch. After the lesion had been delineated using coagulation marks, the endoscope, together with the mounted full-thickness resection device, was inserted into the pouch. Gentle suctioning mobilized the lesion into the cap, and the overthe-scope clip (OTSC) was deployed. Immediate resection using the preloaded snare was then performed. The resection was macroscopically complete. The muscular layer was grasped into the OTSC, thus preserving the integrity of the intestinal wall.

\section{References}

1 Schmidt A, Bauer P, Gubler C et al. Endoscopic full-thickness resection in the colorectum with a novel over-the-scope device: first experience. Endoscopy 2015. DOI: 10.1055/s0034-1391781 [Epub ahead of print]

2 Mannath J, Subramanian V, Singh R. Polyp recurrence after endoscopic mucosal resection of sessile and flat colonic adenomas. Dig Dis Sci 2011; 56: 2389-2395

Bibliography

Dol http://dx.doi.org/

10.1055/s-0034-1392508

Endoscopy 2015; 47: E344-E345

(c) Georg Thieme Verlag KG

Stuttgart · New York

ISSN 0013-726X

\section{Corresponding author}

\section{Christophe Snauwaert, MD}

Department of Gastroenterology and Hepatology Cliniques Universitaires Saint-Luc Université catholique de Louvain Avenue Hippocrate 10

1200 Brussels

Belgium

Fax: +32-2-7648927

christophe.snauwaert@hotmail.com 